Sharif University of Technology
Scientia Iranica
SCIENTIA
I RAN I CA
Transactions A: Civil Engineering
www.scientiairanica.com

\title{
Population capacity threats to urban area resiliency: Observations on chaotic transportation network behavior
}

\author{
M.S. Sharifi, M. Shahabi, E. Abshar, M.H. Khorgami and H. Poorzahedy* \\ Department of Civil Engineering, Sharif University of Technology, Tehran 14588, Iran.
}

Received 10 June 2015; accepted 5 January 2016

\author{
KEYWORDS \\ Urban area resiliency; \\ Population capacity; \\ Transportation \\ network; \\ Chaotic flow; \\ Simulation.
}

\begin{abstract}
This paper is an endeavor to picture excessive population threats to the resiliency of a large metropolitan area. By employing an existing evolutionary model of traffic flow on a small expository network, we show how chaotic situations occur at certain demand and supply parameter values and try to address the questions: How may one determine city population capacity for its transportation network, what happens if the city passes this limit, or how may one return the city to a stable situation when it runs into unstable or chaotic situations. Under certain assumptions, the paper designs a simulation experiment to revisit the phenomenon discovered by Greenshields in 1934, but for a transportation network in a large city. A flow simulation shows that very small changes in the demand and supply of the network result in large variations in the throughput of the network, such that the time series of the latter values to an external observer are chaotic (with positive Lyapunov exponent). The limit to the resiliency of the city from the standpoint of its transportation network capacity is, then, estimated by a value for the city's population. It is then argued that how to take the network out of this situation.

(C) 2016 Sharif University of Technology. All rights reserved.
\end{abstract}

\section{Introduction}

As a material property, resiliency is known as the (utmost) ability to release the energy absorbed when deformed [1]. Resiliency of an ecosystem is defined as the capacity of tolerating external disturbances to remain in the current state, possibly by restoration. Extending this concept to cities, resiliency of a city may be defined as the power to resume the same function after a shock, as it used to perform before the shock. It is a property of absorbing energy surge (shocks), possibly by reorganization and adaptation, without being (appreciably) harmed [1-3]. In this respect,

\footnotetext{
*. Corresponding author. Tel.: +982166019208; Fax: +982166022716

E-mail addresses: porzahed@sharif.edu (H. Poorzahedy); -Siamak.Khorgami@aecom.com (M.H. Khorgami);
}

several characteristics of the social system may help to retain a resilient property. These may include learning, redundancy, adaptability, robustness, resourcefulness, and rapidity $[4,5]$.

There are many reasons why the concept of resiliency is of importance: First, increase in the dimensions of the threats that did not exist before, or increase in the scales of the threats in those dimensions that were previously unimportant or ineffective. These dimensions pertain to the physical, infrastructural, social, and economic, as well as health, and even cultural aspects of these large cities. Second, the rate of effect or speed of change emanating from a shocking event is critical, so it is becoming increasingly hard to cope with these threats in today's larger societies. Third, large cities are increasingly losing ground in their ability to withstand shocks due to their deterioration, high demand (population), and low 
supply of resources and opportunities. Last, but not least, the environment is also becoming increasingly less friendly to large cities (in response to the past and current unfavorable lifestyle of mankind) due to global warming, climate change, and scarcity of energy, as well as alternative energy sources. Thus, large cities are becoming increasingly susceptible to collapse in case of shock emergence, and thereby are in need of redesign and renovation.

Stability of traffic equilibria in day-to-day dynamical systems has received considerable attention in recent years [6,7]. Oscillation of traffic flow is one form of the instability that is focused on by $\mathrm{Li}$ and Ouyang [8], Nie [9], and Xia et al. [10]. It is natural to think of stochasticity and chaos in the framework of traffic flow instability. Flow stability and bifurcation relationship has been studied by Cantarella [11]. Search for chaotic flow in transportation networks came next. Chaotic behavior in a single vehicle-traffic light interaction [12] and two competing vehicles-traffic light interactions [13] was one (micro-level) aspect of the studies on traffic flow. Other studies relate to single facility (for example a freeway section), origindestination demand, and small-size networks [14,15].

Flow dynamics are studied in some details. Lan et al. [16] found strong evidences to conclude that shortterm (one-minute) traffic volume during the morning hours exhibit chaotic, rather than random, phenomena. These evidences are based on wide range comparisons of plots and statistics between the observed one-minute traffic data and their surrogates. They selected some most important indices to establish the parsimony testing procedure, which validated the identification of chaotic traffic time series.

Lin and Lan [17], in examining traffic time series data, conclude that one-minute flow data in the morning hours exhibit chaotic phenomenon, but five- and ten-minute scales do not. Lan et al. [18], employing cellular automaton traffic simulator, study the selforganization formation of traffic. Their experiments on a two-lane freeway show that this phenomenon appears when the density exceeds $45 \%$ (三60 pcu/km). They report for their case, however, that the edge of chaos is at $33.8 \%$ ( $\equiv 45 \mathrm{pcu} / \mathrm{km}$ ), so the ramp metering (traffic control) should start at this level of density to prevent recurrent congestion. A two-stage mathematical model is built by $\mathrm{Xu}$ and Gao [19] to describe the variations of $\mathrm{O} / \mathrm{D}$ flows over discrete time periods (a day or a week). They show that even in a two-dimensional system, chaos phenomena still exist, and find that chaos "propagates" in their two-stage model.

Although long-term traffic forecasting was an important objective in many transportation planning problems up to the 1980 's, it is the short-term flow prediction that basically supports ITS (Intelligent Transportation System) decisions these days. Wang et al. [20] assert that traffic flow data exhibit chaotic properties. They "de-noise" the data using wavelet transform before forecasting, and use phase space reconstruction technique to predict traffic flow reliably in a short-term.

The theory of chaos has also been related to other macro-level concepts such as sustainable transportation $[21,22]$.

The purpose of this paper is to show that there are limits to the resiliency of large urban areas. One such limit is related to the population capacity of the city from the viewpoint of transportation network. The paper considers its contributions to be as follows: (i) Its experiments on small-scale networks are new to the literature; (ii) Its large-scale real-size experimental simulations are also new. It brings in the concept of infrastructural limit to the growth of the city population, which is attempted to be estimated for transportation for the case under study. More importantly; (iii) it experimentally shows how one may bring back a transportation network from a chaotic to a more stable state.

This paper is organized as follows. In Section 2, an existing evolutionary model of traffic flow is described to become the core of the discussion, regarding chaotic flow condition. This discussion is concluded by a way to take the system back from chaotic to normal situation. Section 3 views the transportation network of a large city and designs simulation experiments that initiate the generalization of a freeway section's speedthroughput relationship to a road network, experiencing large variations in a real-size network throughput due to small demand or supply changes. To an external observer, the positive Lyapunov exponent of the time series of the day-to-day throughputs is indicative of the emergence of a chaotic phenomenon.

\section{Evolutionary models of traffic flow}

One type of dynamic traffic flow model is the evolutionary model defined by the following recursive equation [23]:

$$
X^{n+1}=Y\left(X^{n}\right) .
$$

The equilibrium point of the above model may be thought of as the following fixed point:

$$
X^{*}=Y\left(X^{*}\right) \text {, }
$$

which may be defined as the stabilized state of the dynamic system in Eq. (1):

$$
X^{*}=Y\left(\left(\ldots\left(Y\left(X^{*}\right)\right) \ldots\right)\right) \text {. }
$$

Flow stability in dynamic systems has recently been under the scrutiny of many researchers including 
Jin [24]. Jin's model is a continuous model whose fixed point is the well-known user equilibrium flow in the transportation networks [25]. The structure of this model is based on the First-In-First-Out (FIFO) rule violation function. This rule states that for uniform travelers from an origin to a destination, the first one entering the network would be the first one to get out of the network (reaching the destination). Thus, violation of this basic rule is a driving force toward a stable flow. Jin's model is as follows:

Algorithm 1. Jin's Model.:

- Step 1. Consider a feasible flow in the network traversing the existing paths from origins to destinations. Specify the model parameter, $\lambda$;

- Step 2. Compute the travel times $\left(t_{p}^{k s}\right)$ of all the used paths $p$ (with positive flow) for $\mathrm{O} / \mathrm{D}(k, s)$;

- Step 3. Compute the FIFO violation of path $p$ for $\mathrm{O} / \mathrm{D}(k, s)$ as:

$$
J_{p}^{k s}\left(x_{p}^{k s}\right)=d^{k s} \cdot x_{p}^{k s} \cdot\left(t_{p}^{k s}-v^{k s}\right) .
$$

If the FIFO functions are zero, Goto Step 5; otherwise, continue.

- Step 4. Update the path flows as follows:

$$
x_{p}^{k s}(\tau+1)=x_{p}^{k s}(\tau)-\lambda^{k s} \cdot J_{p}^{k s}\left(x_{p}^{k s}(\tau)\right) .
$$

- Step 5. End. The system has reached equilibrium.

In Step 3 of the above algorithm, $v^{k s}$ is the weighted average of the different path travel times:

$$
v^{k s}=\sum_{p \in W^{k s}}\left(t_{p}^{k s} \cdot x_{p}^{k s}\right) / \sum_{p \in W^{k s}} x_{p}^{k s} .
$$

It can easily be shown that by embedding Eq. (6) in Eq. (4), the FIFO violation function will turn out as:

$$
J_{p}^{k s}\left(x_{p}^{k s}\right)=x_{p}^{k s} \cdot \sum_{q} x_{q}^{k s}\left(t_{p}^{k s}-t_{q}^{k s}\right) .
$$

In fact, Eq. (5) in Step 4 of the algorithm is a linear adaptation of flow, in which the change in path flow, $\dot{x}_{p}^{k s}$, equals $-J_{p}^{k s}\left(x_{p}^{k s}\right)$ :

$$
x_{p}^{k s}(\tau+1)=x_{p}^{k s}(\tau)+\lambda^{k s} \cdot \dot{x}_{p}^{k s}(\tau) .
$$

Parameter $\lambda^{k s}$ represents the sensitivity of the network users in responding to the current differences in their path travel times with the current respective $\mathrm{O} / \mathrm{D}$ average path travel time value, and adjusts the current path flows to bring the path travel times close to the respective $\mathrm{O} / \mathrm{D}$ average value.

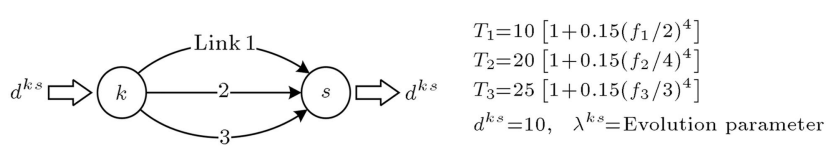

$$
\begin{aligned}
& \text { Example FIFO violation function for path } 1 \text { : } \\
& \begin{array}{r}
J_{1}^{k s}=f_{1}\left\{\left[10\left(1+0.15\left(f_{1} / 2\right)^{4}\right)-20\left(1+0.15\left(f_{2} / 4\right)^{4}\right)\right] f_{2}+\right. \\
\left.\left[10\left(1+0.15\left(f_{1} / 2\right)^{4}\right)-25\left(1+0.15\left(f_{3} / 3\right)^{4}\right)\right] f_{3}\right\}
\end{array} \\
& \text { Example of flow evolution function for path 1: } \\
& f_{1}^{k s}(t+1)=f_{1}^{k s}(t)-\lambda^{k s} \cdot J_{1}^{k s}(t)
\end{aligned}
$$

Figure 1. The example network and its link specification functions.

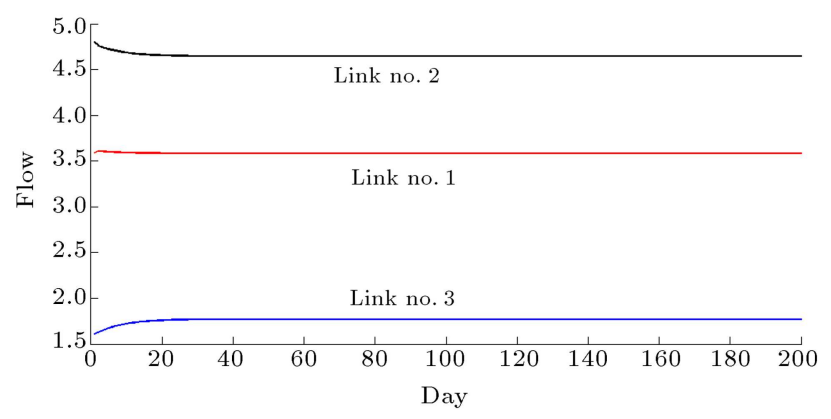

Figure 2. Path flow evolution over the iterations (days) of the algorithm.

\subsection{An example network}

Let us study the simple example network of Sheffi [25], as shown in Figure 1, by the Jin's algorithm mentioned above. The network has one $\mathrm{O} / \mathrm{D}$ pair with demand $d^{k s}=10$. The link travel time functions and the expressions for the FIFO violation functions of the $\mathrm{O} / \mathrm{D}$ paths are shown in this figure, where each link is an O/D path. Suppose we start the algorithm by the initial solution of $(3.39,5.0,1.61)$. We may, then, compute the next time (day) flow vector by the following set of equations:

$$
x_{l}(\tau+1)=x_{l}(\tau)-\lambda J_{l}(\tau), l=1 \text { to } 3 .
$$

Suppose $\lambda=0.002$. Figure 2 shows the evolution of the path flows over the iterations of the algorithm and its convergence to the point of $x^{*}=(3.59,4.70,1.71)$. This is the User Equilibrium (UE) flow in the network, with the corresponding travel time vector of $t^{*}=$ $(25.57,25.72,25.40)$, approximately the same for the three paths.

Let us increase $\lambda$ to 0.005 . Figure 3 shows that the solution does not converge to a specific point, and the process alternates between two solutions (i.e., it bifurcates). We increase $\lambda$ further to 0.0064 . Figure 4 depicts the behavior of the flow in path (link) 1 , which is rather "chaotic" (i.e., the "daily" traffic flow in the path wonders around, taking many values). Similar behavior exists for the other two links.

Figure 5 shows the variation in the flow of link $1, x_{1}$, as $\lambda$ increases from $1.0 \times 10^{-3}$ to $6.4 \times 10^{-3}$ (Similar variations happen for $x_{2}$ and $x_{3}$, the flows in 


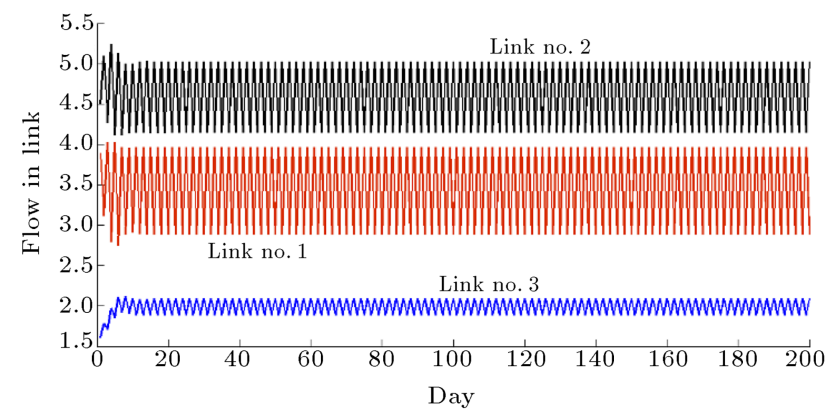

Figure 3. Alternating path flows in the example network (bifurcation).

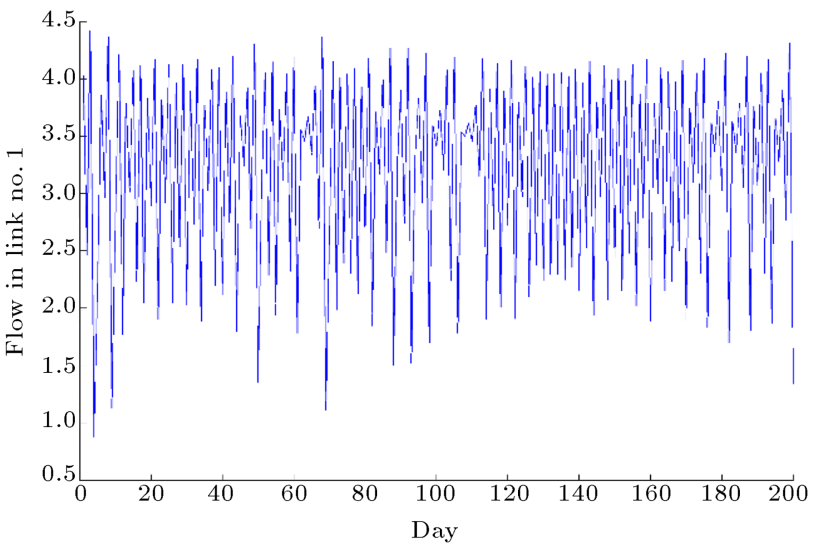

Figure 4. Chaotic path flows in the example network.

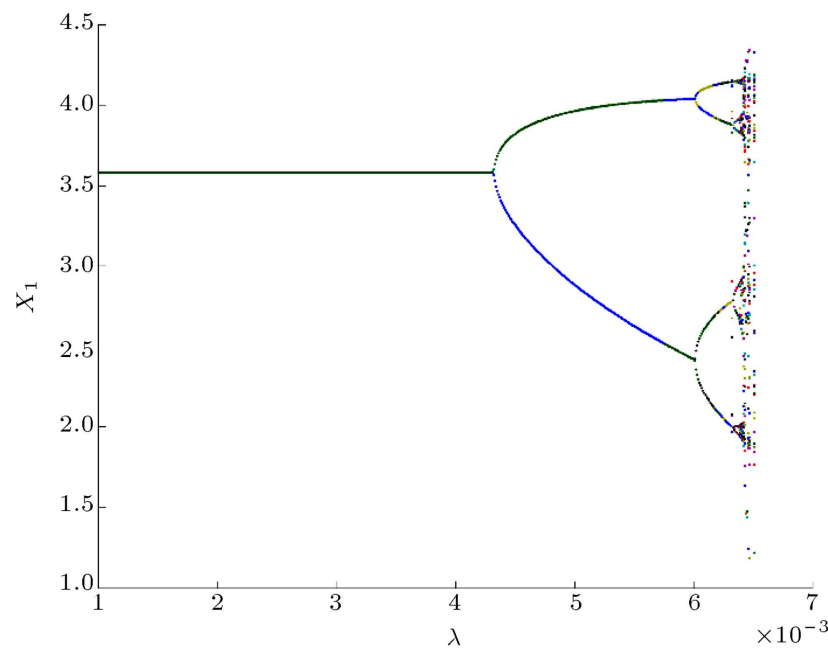

Figure 5. Equilibrium, bifurcation, and chaotic flows in path 1 for various values of $\lambda$.

paths (links) 2 and 3 , respectively.) For $\lambda=1 \times 10^{-3}$ to $4.5 \times 10^{-3}$, the flow in path 1 tends to the UE flow. As $\lambda$ increases further, the flow value in link 1 bifurcates (several times) until $\lambda=6.4 \times 10^{-3}$, where it enters into a chaotic state. In such a state, the users experience various levels of travel times each day. However, Figure 5 shows that the flow in path 1 is contained within a limited range. Figure 6 shows this variation of path flow vector with $\lambda$ in the three dimensions of

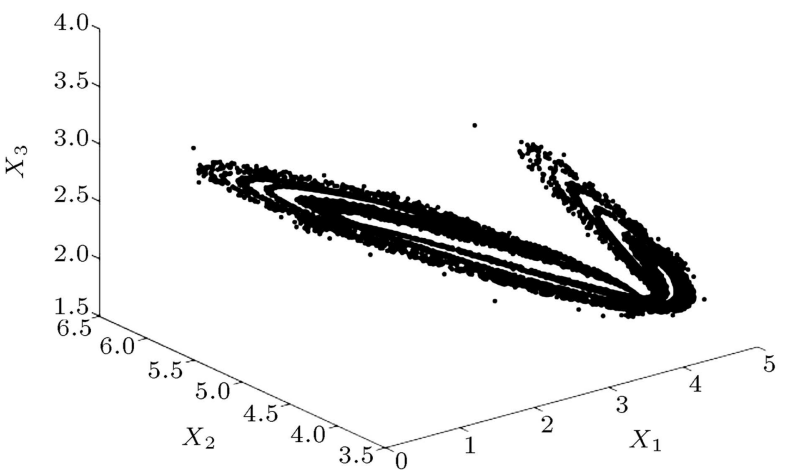

Figure 6. The attractor space of path flows for the three-link example network.

$\left(x_{1}, x_{2}\right.$, and $\left.x_{3}\right)$. The latter is the attractor space of the path flows (a "galaxy" of the solutions).

\subsection{The need for uncertainty analysis in the problem}

One common assumption in most network flow problems is the determinacy and constancy of the parameters of the problem, such as (O/D) demand. For the latter assumption, the flow levels are computed for the expected levels of demand. The analysis of the problem is usually done with the assumption that the expected values of effectiveness measures equal the respective measures with the expected values of the parameters. However, this assumption is correct only for the linear systems, while the flow problem in a network is a nonlinear one. According to the Jensen's inequality [26,27], if $f(x)$ is a convex function and $x$ an uncertain input, then:

$$
E[f(x)] \geq f(E[x])
$$

Put in an example, the total travel time of the users of the network under (constant) expected demand input will underestimate the actual sum of the total user travel times. This shows that consideration of uncertainty in network flow problems leads to better analyses of these problems, and hence better decisions.

Uncertainties in network supply parameters are as important as those in demand side. Variations in the weather conditions, traffic incidents, road/lane closures for maintenance, etc. have significant effects on the operation and effectiveness of the network. Analyses of networks without due uncertainty considerations may only be a simplification of the realities. Nevertheless, in most network analyses, the supply functions, such as link travel time functions, are considered known and invariant. As an example conclusion, if supply and demand have their own uncertainties, say probability distributions, one may only be confident in reliable operation of the system when supply exceeds the demand. This level of reliability (i.e., the probability that supply exceeds the demand) is different from 
the reliability of the system when the expected value of the supply exceeds the expected value of the demand [28].

\subsection{Behavior of the example network under supply uncertainty}

Consider, once again, the example network, but this time with more general link travel time functions as follows:

$$
\begin{aligned}
& t_{1}=10\left[1+0.15\left(\frac{x_{1}}{2 / \rho}\right)^{4}\right], \\
& t_{2}=20\left[1+0.15\left(\frac{x_{2}}{4 / \rho}\right)^{4}\right], \\
& t_{1}=25\left[1+0.15\left(\frac{x_{3}}{3 / \rho}\right)^{4}\right],
\end{aligned}
$$

where $\rho \geq 1$ is a random factor representing all detrimental effects on practical capacities of the links, with $\rho=1$ representing the original (base) network. Let us now apply Jin's model to this case for $\lambda=$ $1.5 \times 10^{-3}$ and $d^{k s}=10$, which has one fixed point, i.e. the UE flow. The results are shown in Figure 7. As is evident in this set of figures for the three paths in the network, for $0 \leq \rho<1.6$ the network possesses stable (UE) flow. The flow levels bifurcate in the range of $1.6 \leq \rho<1.7$, and then turn into a chaotic state in the range of $1.7 \leq \rho<1.72$.

Increasing the demand, for example from $d^{k s}=10$ to 25 , would shorten the range of the same type of evolution for the same value of $\lambda$, as shown in Figure 8.
As is evident from this figure, the inflection points from stable flow to bifurcation, and from bifurcation to chaotic, have changed from 1.6 to 1.045 and from 1.7 to 1.065 , respectively. The total range of these changes has reduced from $[1.0,1.72]$ to $[1.0,1.0655]$, which is a significant change. That is, the network would become more susceptible to failure (get into a chaotic state) when the demand for travel increases (flows get closer to the practical capacities of the links).

\subsection{Behavior of the example network under demand uncertainty: Population capacity}

Determination of the city's population capacity (e.g., from the viewpoint of its transportation infrastructure) is an intriguing problem to city planners and authorities. What is the population level in the city whose transportation infrastructure can support at an acceptable level of service?

Let us pose this question in the framework of our example network. This time, we increase the demand, $d^{k s}$, by multiplying it by a factor $k, k \geq 1.0(k=1.0$ represents the original/base network). Figure 9 shows the results of the application of Jin's model on our example network for $\lambda=1.0 \times 10^{-3}$ and initial demand $d^{k s}=10$, which has one fixed point; a UE flow. This figure shows that starting from $k=1.0$, the flow level remains stable up to $k<1.55$. The network path flows enter into the bifurcation phase at $k=1.55$ and remain in this state until $k<1.65$, at which point they enter into the chaotic condition, which lasts until $k<1.655$. Thus, we might say that the network can handle an $\mathrm{O} / \mathrm{D}$ demand up to $1.55 \times 10=15.5$ units. For a trip generation rate of 3 units of trips/unit of population,
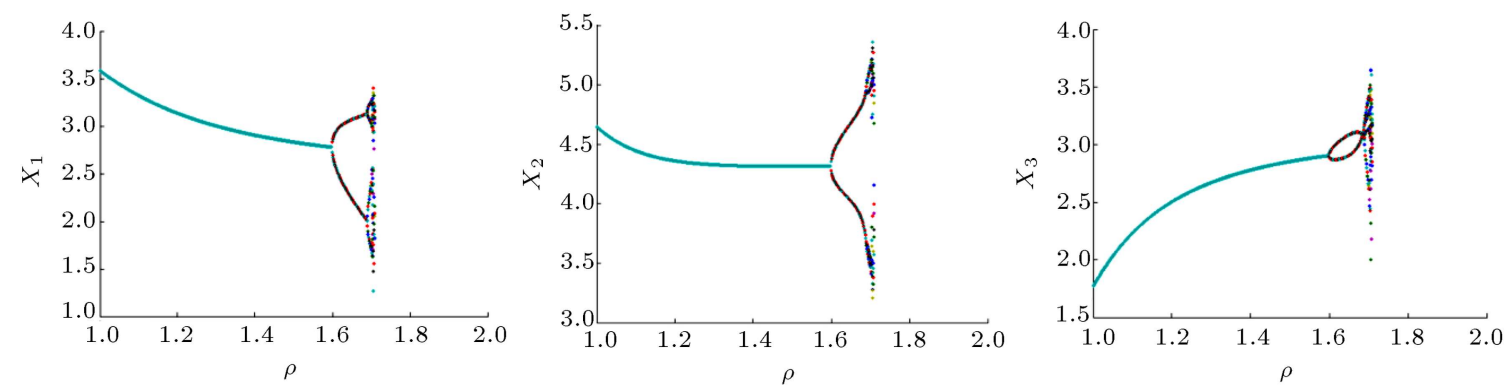

Figure 7. Variation of path flows for different values of $\rho\left(\lambda=0.0015\right.$ and $\left.d^{k s}=10\right)$.
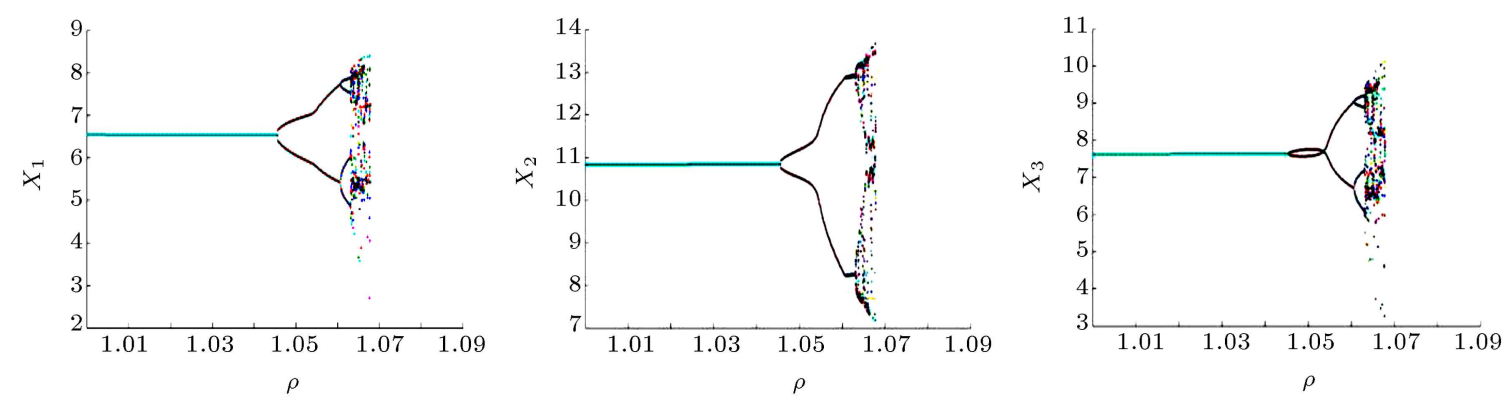

Figure 8. Variation of path flows for different values of $\rho\left(\lambda=0.0015\right.$ and $\left.d^{k s}=25\right)$. 

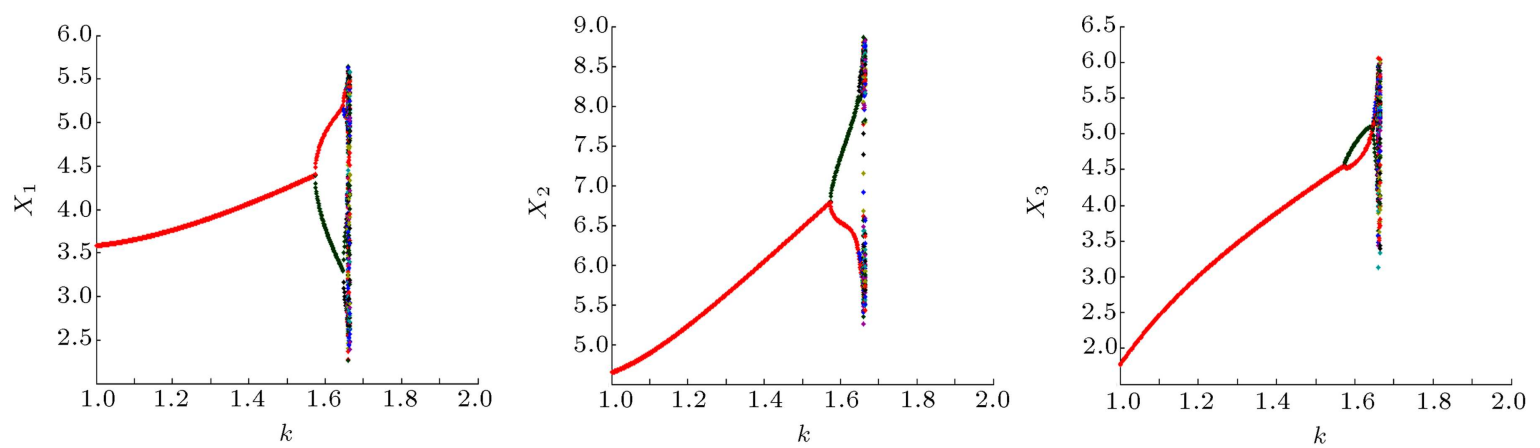

Figure 9. Variation of path flows for different values of $k\left(\lambda=0.001\right.$ and $\left.d^{k s}=10 k\right)$.
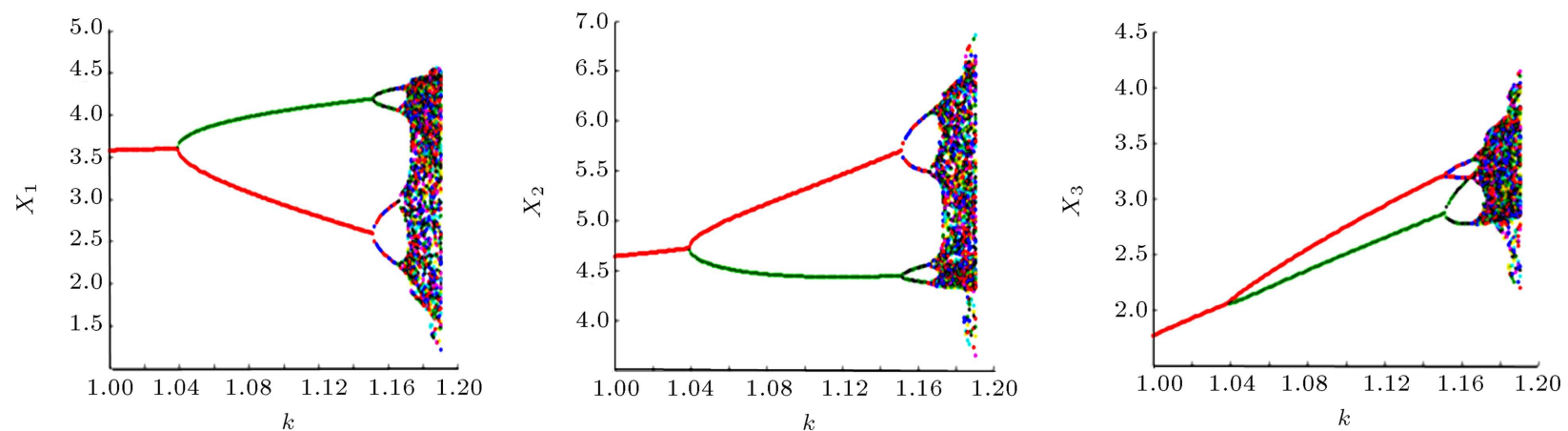

Figure 10. Variation of path flows for different values of $k\left(\lambda=0.0032\right.$ and $\left.d^{k s}=10 k\right)$.

population capacity of the city for this network is about $46(\approx 3 \times 15.5)$ units.

Suppose, now, that the sensitivity of the demand for transportation increases from $\lambda=1.0 \times 10^{-3}$ to $3.2 \times 10^{-3}$. Running the model, once again, with this new parameter value, would result in Figure 10. This figure shows that the flow stability range will diminish to $1.0 \leq k \leq 1.04$ (previously, 1.55). The state of flow bifurcation happens in the shorter range of $1.04 \leq k<$ 1.17 (previously, 1.65). Finally, the state of chaotic flow happens in the range of $1.17 \leq k<1.19$ (previously, 1.655). The reader will note that with the higher value of $\lambda$ (higher user reaction), the network falls into unfavorable states with a much smaller increase in demand.

\subsection{Counteracting chaotic situation}

Control of chaos, and avoiding its occurrence, is one important aspect of dynamical systems, as is also emphasized by $\mathrm{Xu}$ and Gao [19]. To avoid chaotic situations, one may make sure that the parameters of the system are in the respective ranges of stable values. However, the answer to the more serious question of 'how can one take a chaotic system into a (more) stable situation?' is not straightforward. Let us, once again, consider the three-link network in Figure 1 and run Algorithm 1, but this time with the following timedependent parameter:

$$
\lambda=0.0067-1.8 \times 10^{-5} \tau .
$$

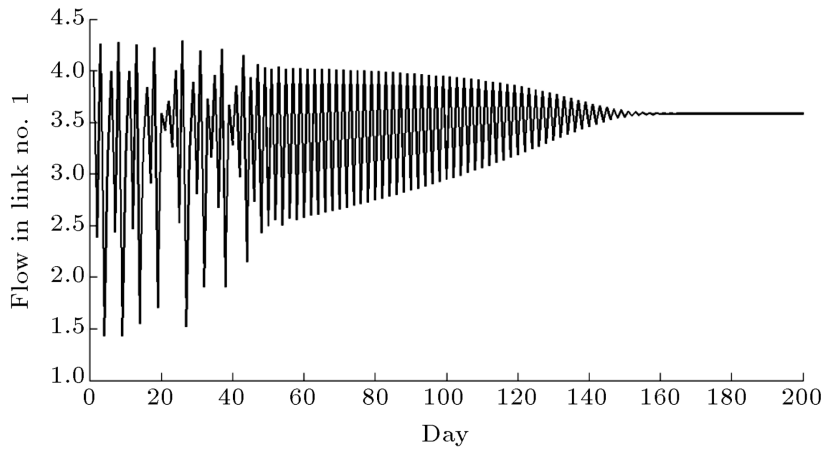

Figure 11. The flow in path 1 for adaptive value of $\lambda=0.0067-1.8 \times 10^{-5} \tau$.

Note that the system is again totally deterministic and that at $\tau=0$, we have $\lambda=0.0067$, which is in its chaotic range. Figure 11 shows the value of flow in link 1 for different time intervals. It shows how the time adaptability of $\lambda$ brings the system (the link flows) back to the stable UE solution shown in Figure 2.

\subsection{Summary of Section 2}

The concept of chaos in deterministic nonlinear systems, as discussed above, represents a state of disorder and irregularity for a system of difference equations, as given in Eq. (8), defining the time-dependence of path flows in the network. More specifically:

$$
X^{n+1}=Y\left(X^{n} \mid \lambda\right)
$$

We find that under certain conditions, flows reach 
stable values or a fixed point. Variation (increase, here) of an exogenous parameter doubles the fixed points at particular values and this process continues until the fixed points become infinite in number, while the parameter values are all finite. In fact, we noted that these large changes in the number of fixed points followed very small changes in the parameters, all being within rather short intervals.

Later, in two other experiments with the example network, we considered the uncertainty in the link practical capacity (by a new parameter, $\rho$ ), as well as the uncertainty in the $\mathrm{O} / \mathrm{D}$ demand (considered through a new parameter, $k$ ). The Jin's model has been started in a state which had a unique UE fixed point with its original parameter value $\lambda$ at $k=\rho=1.0$. We observed that changes in the new parameters ( $\rho$ and $k$ ) in the direction of lower link capacities or higher demand would lead the flow system into a chaotic state much faster (that is, at much tighter ranges of $\lambda$ ). Similar observations are made with larger networks $[29,30]$.

\section{Simulation model of network flow}

We consider, now, an actual network of a large city and change the capacity of the system by link removals that constitute only negligible changes in the network total Lane-Kilometers (LK) and similar (same order) changes in the $\mathrm{O} / \mathrm{D}$ pair demands. We will show that the response of the system to these changes will be significantly magnified.

The system of concern is the network of a real case, the city of Mashhad, Iran, with a population of 2.3 million at the time of the study. An aggregate network of this city is made of 872 nodes and 1184 links; 15 (super-)sources and (super-)sinks represent the 141 traffic zones of the study area. Figure 12 shows this network.

The set of models that estimate the demand is of four-step type, which is based on extensive origin/destination survey results and databases that were conducted in 1994. There are seven trip purposes, for each of which there is a pair of trip production and attraction models, each being a function of several (zonal) variables such as employment level, car-ownership, and student number.

Trip distribution is made by trip purpose using doubly constrained Fratar type models:

$$
T_{i j}^{p}=\alpha_{i}^{p} \beta_{j}^{p} O_{i}^{p} D_{j}^{p} d_{i j}^{\gamma^{p}}
$$

where $T_{i j}^{p}$ is the total $\mathrm{O} / \mathrm{D}$ trips from origin $i$ to destination $j$ for the trip purpose $p, O_{i}^{p}$ is the total trips produced from zone $i$ for trip purpose $p, D_{j}^{p}$ is the total trips attracted to zone $j$ for trip purpose $p, \alpha_{i}^{p}$ and $\beta_{j}^{p}$ are two balancing factors for the trip purpose $p$ that are

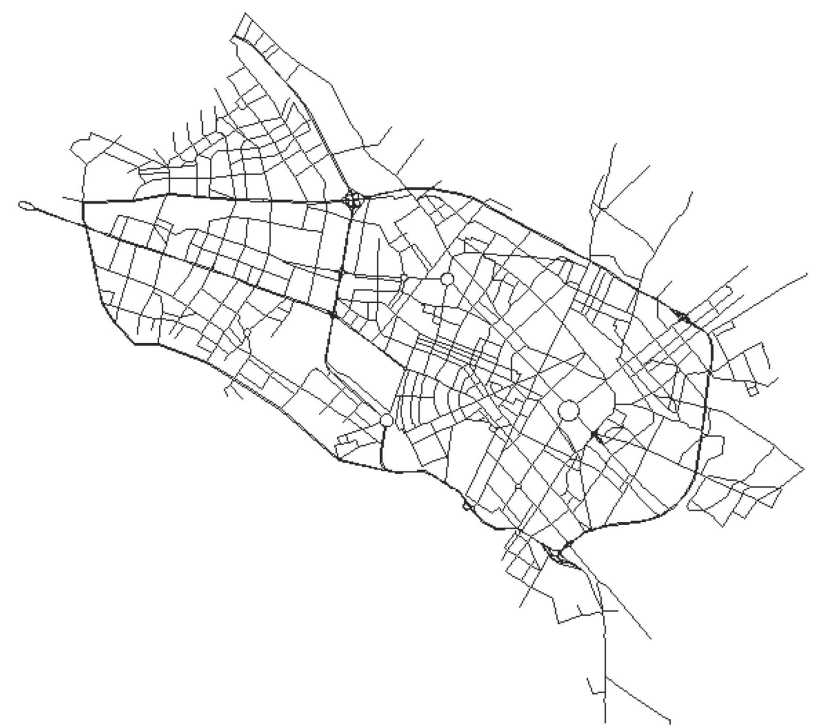

Figure 12. The network of the city of Mashhad.

dependent on origin $i$ and destination $j$, respectively, and $\gamma^{p}$ is a model parameter for the trip purpose, $p$. The model is subject to two constraints:

$$
\sum_{j} T_{i j}^{p}=O_{i}^{p}, \forall i, \text { and } \sum_{i} T_{i j}^{p}=D_{j}^{p}, \forall j,
$$

with $\sum_{i} O_{i}^{p}=\sum_{j} D_{j}^{p}$. These models are calibrated by successive row-column balancing for each trip purpose $p$.

The mode choice model for trip purpose $p$ is multinomial logit, as follows:

$$
p_{i j}^{m, p}=e^{u_{i j}^{m \cdot p}} / \sum_{k \in M} e^{u_{i j}^{k} \cdot p},
$$

where $p_{i j}^{m, p}$ is probability of choosing mode $m$ among the set of alternative modes $M(m \in M)$ in a trip from zone $i$ to zone $j$ for purpose $p$; and $u_{i j}^{m}$. $p$ is utility of mode $m$ for this trip, which is a function of the characteristics of passenger, origin, destination, and mode of travel, as follows:

$$
u_{i j}^{m \cdot p}=\sum_{l} \alpha_{l}^{m \cdot p} X_{i j, l}
$$

where $\alpha_{l}^{m . p}$ is a coefficient of the $l$ th variable of the utility function, $X_{i j, l}$, for trip purpose $p$, which may be either mode-specific or generic. The set of available modes $M$ includes two-wheeled vehicles, private cars, taxi, and bus for all trip purposes with an additional minibus mode for school trips. Variables $X_{i j, l}$ include car-ownership and travel time for cars, two-wheeled vehicle ownership and O/D distance for this type of vehicles, in-vehicle travel time, out-vehicle travel time, and number of times passengers board buses.

Traffic assignment model consists of an optimal strategy procedure [31], which assigns public transport 
passengers to its network, and a multi-class user equilibrium flow, which assigns the demand for the other five vehicles to the road network [25]. Road network assignment considers delays at signalized and un-signalized intersections, turn prohibitions, and six types of road volume-delay functions. The public transport assignment considers several vehicle types in public transport system. The interaction between public and private modes of transportation is also considered by the model.

The model is coded in EMME/2 environment [32] to estimate the (hourly) demands, assign them to the network, and estimate the operational characteristics of the network. These characteristics include vehiclekilometers, vehicle-hours, average vehicle/passenger speed in the links and in the networks, network congestion levels and the respective distribution, different fuel consumptions, and different emissions of pollutants. These are available by demand type (person/vehicle trips), network type (public/private), vehicle type, road type, and geographical region [33].

\subsection{Experimental design}

The purpose of the following experiment is to simulate the flow over the network in a particular hour of the day over different days. The system of concern is the network of a large city. We assume the following:

(a) In a given day, demand varies over time from 00:00 to $24: 00$, and we assume that the hourly variation of a typical/mean demand over the network is known by an O/D study (mentioned above). However, to simulate a case similar to an existing well-known experiment by Greenshields [34], we vary demand to create cases $n=0,1, \ldots, N$ with $d^{k s, n}=c . d^{k s}$ to be the $(k, s)$ element of the $\mathrm{O} / \mathrm{D}$ matrix, where $c=c_{0}+c_{1} . n$ for $c_{0}$ and $c_{1}$ being two constants. In passing, we note that a similar concept has been employed to find the reserve capacity of a network in other studies (see, e.g. $[28,35])$;

(b) Given the O/D demand and the network, distribution of the flow over the network is a deterministic procedure. For a given hour of the day, say the peak hour, the flow over the network varies dayby-day. One source of this variation is small changes in the timing of the (fixed) demand appearing in the network. We assume a normal distribution for this variation with known mean (mentioned in (a)) and a standard deviation equal to a small fraction of this mean. Let $x^{k s}$ be the total O/D demand from origin $k$ to destination $s$ during a given time period, and that:

$$
x^{k s}=d^{k s}+\sigma^{k s} Z,
$$

where $Z \sim N(0,1)$. Then, $x^{k s} \sim N\left(d^{k s}, \sigma^{k s}\right)$. Banks et al. [36] present a procedure for generating standard normal random numbers by two independent uniformly distributed random numbers in interval $[0,1]$. For the purposes of this study, we assume that $\sigma^{k s}=0.1 d^{k s}$, for all $(k, s) \in W$, where $W$ is the set of $\mathrm{O} / \mathrm{D}$ pairs;

(c) Another source of variation of the daily flow in the network is small variation in the supply over the network due to: (i) operational factors (lane or road closures due to vehicle accidents, road repair, unauthorized stops and parking, and bus stops in front of a column of moving vehicles in a lane); (ii) weather-related factors (rain fall, water ponds, icy pavement, and low visibility due to fog or rain); and (iii) human-related factors (slow-moving and badly-maneuvering vehicles).

For random variation of supply, we assume, for simplicity, that the major incident in road capacity reduction is traffic accident, and that if it happens in a link, it closes the link completely for that time period under considerations. It is assumed that accidents occur in the links of the network independent from each other, proportional to the vehicle-kilometers of travel in that link, but dependent on the type of the link $k$ (expressway, $e$, and arterial, a). Then, we may write:

$$
p_{a}=\left(l_{a} \cdot x_{a}\right) /\left(\sum_{a \in A} l_{a} \cdot x_{a}\right)
$$

where $p_{a}$ is the probability of an accident occurrence in link $a, x_{a}$ is the passenger car (equivalent) volume of traffic, and $l_{a}$ is the length of this link. Each link $a$ has a type $k$. Suppose, also, that accident occurrence in the network for each link type $k$ is a stochastic process, $\left\{N_{t}^{k} ; t \geq\right.$ $0\}$, following a Poisson rule with average rate of arrivals of $\lambda^{k}$ for all links of type $k$. Assume $\lambda^{a}=6$ accidents per hour (of the time period of concern) for $618 \mathrm{~km}$ of arterials, and $\lambda^{e}=2$ for $162 \mathrm{~km}$ of expressways, per day. Then, the probability of $n^{k}$ accidents in a unit time interval $(t=1 h r)$ may be computed as:

$$
\begin{aligned}
p\left\{N_{t}^{k}=n^{k}\right\} & =\left(e^{-\lambda^{k} \times 1}\left(\lambda^{k} \times 1\right)^{n^{k}}\right) /\left(n^{k} !\right) \\
& =e^{-\lambda^{k}}\left(\lambda^{k}\right)^{n^{k}} /\left(n^{k} !\right) \\
k & =a, e .
\end{aligned}
$$

(d) Observations in the city under study show that link disruption, due to an accident occurrence in the link, takes about $1 \mathrm{hr}$ before clearance of the accident scene. We assume that accidents occur at the beginning of the time period under study and that users become aware of the accident locations 
by local media and change path choices to their destinations to form the new user equilibrium flow. The latter assumption may be plausible by the virtue of repetition of the urban trips, particularly the morning work and school trips which are basically mandatory. Furthermore, since the changes in demand and supply are marginal (as we will see later in this paper), the difference between the effects of these assumptions and the actual phenomena is considered marginal to the higher orders, and hence ignored for simplicity, without causing appreciable changes in the results.

The performance of the system changes as a function of the state of demand and network in each day. Let us define the following:

Definition 1. $(k, s)$ demand is responded satisfactorily if $t_{u}^{k s} / t_{0}^{k s} \leq \tau$, where $t_{u}^{k s}$ and $t_{0}^{k s}$ are, respectively, the user equilibrium in time period $u$ and the free flow travel time from origin $k$ to destination $s,(k, s) \in W$, and $\tau$ is a real number showing the minimum expected level of service (for example, $\tau=30$ ). The subscript $u$ is the time period under consideration (e.g. the morning peak hour period).

Definition 2. The throughput of the network during a time period $u$ under study (e.g., peak hour) is the sum of all satisfactorily responded demands, $q_{u}=$ $\sum_{(k, s) \in W} \cdot x_{u}^{k s} \cdot z_{u}^{k s}$. In this relation, $z_{u}^{k s}=1 / 0$ if in time period $u$ the demand for $(k, s) \in W$ is responded satisfactorily or not.

\subsection{Network flow simulation algorithm}

The following procedure has been devised to simulate traffic flow in the city under study:

- Step 0 (Preparation). Obtain the O/D flow matrix $\left(d^{k s}\right)$. Define a practically absolute number of accidents for each road type $k$ for Monte Carlo simulation. Set iteration number, $m:=1$;

- Step 1 (Identifying the number and place of traffic accidents in the network for the time period in the next day). Use Eq. (20) to specify the number of accidents for the time period in the next day (e.g., morning peak hour), and Eq. (19) to specify the $\operatorname{link}(\mathrm{s})$ in which these accidents occur, for each road type $k$, by Monte Carlo simulation. Omit these links from the original network and specify the currently available network;

- Step 2 (Equilibrium flow computation). Compute the new $\mathrm{O} / \mathrm{D}$ demand by Eq. (18), finding the standard normal random variable $Z$ and assuming $\sigma^{k s}=0.1 d^{k s}$ for all $(k, s) \in W$. Then, compute the user equilibrium flow by assigning this demand to the currently available network in Step 1;

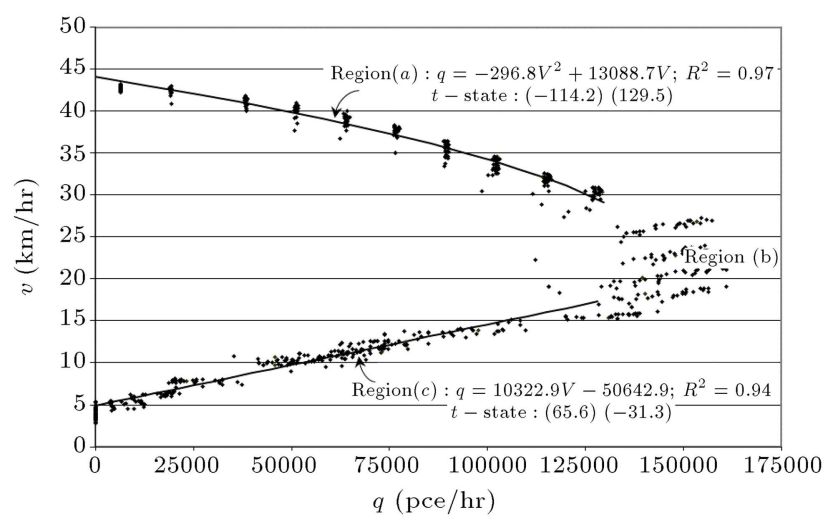

Figure 13. The speed $(V)$-throughput $(q)$ points for 30 runs for each of the 23 demand cases for the city of Mashhad.

- Step 3 (Computation of network performance). Compute the total network throughput according to Definitions 1 and 2, and the network average speed as $V=$ (total vehicle-kilometers)/(total vehicle-hours);

- Step 4 (Stopping criterion). If $m \leq M$ (say, 30), STOP; otherwise, set $m=m+1$ and Goto Step 1 .

\subsection{Network flow simulation results}

In order to see the behavior of the network under the load of varying $\mathrm{O} / \mathrm{D}$ demand, the algorithm in Section 3.2 has been run for $M=30$ times for each of the demand cases $n=0,1, \ldots, N$. Assume $c_{0}=0.1$, $c_{1}=0.1$, and $N=23$. Figure 13 shows the resulting $V-q_{u}$ points in this coordinate space.

The reader may note the resemblance of the kind of relationship in this figure with that of the Greenshields' observations for a freeway section (see [37]). Three distinct regions may be seen in Figure 13. These are Regions (a) and (c) with more or less clear relationships between $V$ and $q$, as shown in this figure. The statistically estimated mathematical relationships are shown beside the respective curve/line. The parameters of these functions are statistically significant above $99 \%$ confidence level, and the $R^{2}$ 's of the regression are 0.97 and 0.94 for the functions of Regions (a) and (b), respectively.

Next, we see Region (b), which has spread over points in the coordinate space. This is the same message conveyed by the Greenshields' observations for a freeway section. Increase in the incoming flow (characterized by $n$ in our experimental procedure, as discussed in assumption (a) in Section 3.1) would increase the density of the flow, which in turn reduces the speed. This is true for the whole $M=30$ observations that form a bunch of close points in Figure 13. This reduction in the level of service is accompanied by the increase in the rate of the throughput, until a point is reached where this rate becomes a maximum. At this point, the process ceases to retain a clear relationship between the state variables 


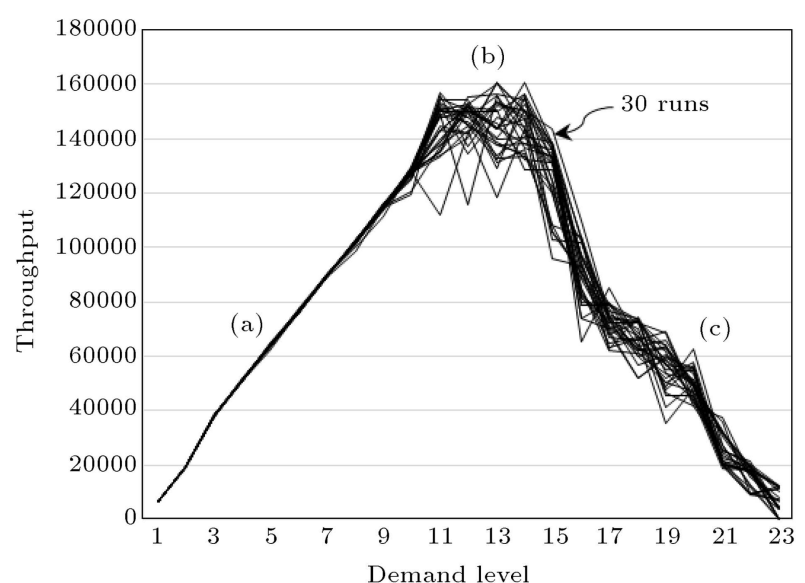

Figure 14. Variation of the throughput for 30 runs of the algorithm for 23 demand cases.

speed and throughput, or flow (and presumably the flow density in the network), so that a turbulence (or instability, or "chaotic" situation) occurs. From this point on, increase in the incoming flow (and its density) would reduce the speed further, while reducing the throughput rates as well, which together bring the traffic to a standstill situation.

Figure 14 shows the trajectory of the throughput in the $M=30$ runs (i.e., days) of the algorithm for each of the 23 demand level increases. It shows this variation for Region (a) and the other two regions, respectively. The reader notes that the throughput in Region (a) over the days is rather stable. However, this flow is turbulent in Region (b), with this characteristic diminishing as traffic goes to a standstill condition in Region (c).

\subsection{Chaotic interpretation of the day-to-day fluctuation of the network throughput}

Chaos is a phenomenon that may occur in systems which are dynamic, nonlinear, sensitive to their parameters, sensitive to their initial conditions, or possess self-similarity [21,38-41]. Schuster and Just [42] present several possible qualitative criteria for chaotic notions: "looking chaotic," exhibiting broadband noise, decaying correlation function, and showing space filling points in Poincaré map. They also introduce some quantitative measures to characterize the case of deterministic chaos, such as Lyapunov exponent, invariant measure, and correlation functions, for one-dimensional Poincare' maps, as well as quantitative measures for other cases.

Consider the following model of user equilibrium flow:

$$
\left(x_{i j}\right)=\operatorname{Assign}\left(D\left(d^{k s}(\alpha)\right), N(V, A(\beta))\right) .
$$

This model is an abstract form of the following problem:

$$
\begin{aligned}
& \operatorname{Min} U(x)=\sum_{(i, j) \in A(\beta)} \int_{0}^{x_{i j}} t_{i j}(u) d u, \\
& \text { s.t. : (i) } \sum_{p \in P_{k s}} x_{p}^{k s}=d^{k s}(\alpha), \forall(k, s) \in W, \\
& \text { (ii) } x_{p}^{k s} \geq 0, \forall p \in P_{k s}, \forall(k, s) \in W, \\
& \text { (iii) } x_{i j}=\sum_{(k, s) \in W} \sum_{p \in P_{k s}} x_{p}^{k s} \cdot \delta_{i j p}^{k s,}
\end{aligned}
$$

where $\alpha$ and $\beta$ are, respectively, the ratio of the total $\mathrm{O} / \mathrm{D}$ demand appearing in the network, and the ratio of the total length of the links available to the users of the network, over the long run average value of the respective quantities, at the time period $u$ of consecutive days.

Time series analysis of data is a prevalent method to verify chaotic behavior of complex systems $[43,44]$. We, also, take advantage of this method in our study of large real-size transportation network. Consider, now, another experiment in which 300 observations are made in Region (b) of Figure 13. (There are about 300 working days in a year.) Figure 15 shows a schematic representation of the results of this experiment. There are two time dimensions running in this experiment; the day time which starts early in the morning till the peak flow time in the network, when it operates at the capacity level. Then, there is another time running on the day-to-day basis. To an external observer, the result would be the maximum throughputs obtained in each day, whose fluctuation is shown in the lower-right part of Figure 15.

Figure 16(a) and (b) show the variations of the parameters $\alpha$ and $\beta$ for the 300 runs of this experiment, respectively. They show that these variations are very small. Figure 16(c) depicts the ratio of the maximum throughput of the day over the average value of these 300 runs (days). This part clearly shows a large variation for the small changes in the input parameters. To the external observer, this is a time series data which has a positive Lyapunov exponent of 0.0236, showing that the series is chaotic (see Schuster and Just [42], and also Best et al. [45], who employ Lyapunov exponent for flight control systems as a warning onset of instability).

\subsection{The city network capacity}

We raise, once again, a similar question that was posed in Section 2.5: What is the capacity of the city network? We define the capacity as being the maximum demand that the network may handle without falling into a "chaotic" situation. Let us answer this question in an overall and informal manner.

Suppose $q_{\max }$ is the total city transportation demand (in car equivalent per hour) before it takes 


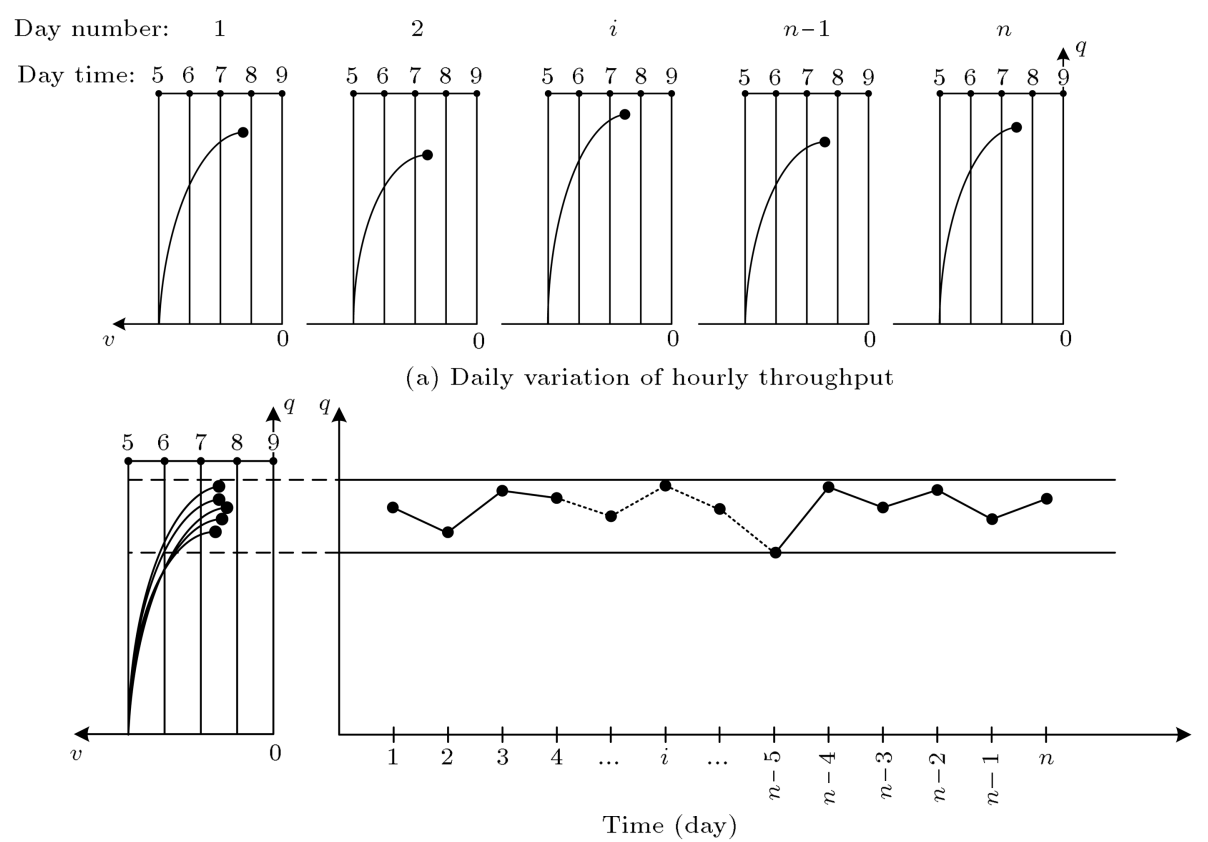

(b) Maximum throughput time serious

Figure 15. A schematic representation of day-to-day maximum throughput evolution.

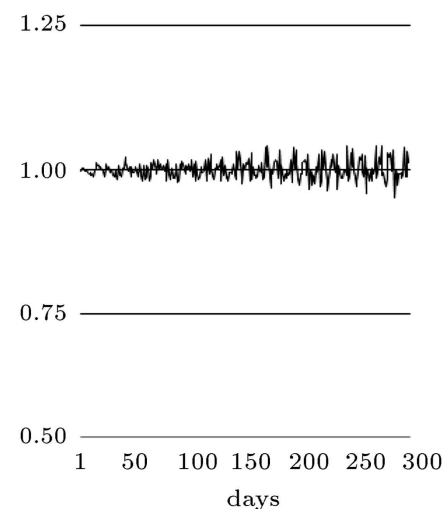

(a) Total O/D demand over average value of this quantity

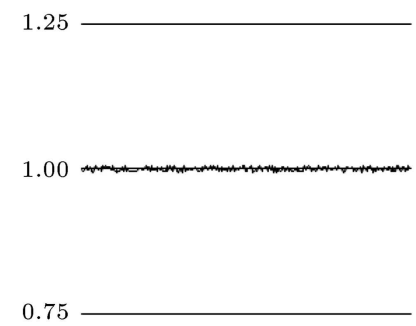

$0 . 5 0 \longdiv { 1 } \quad 5 0 \quad 1 0 0 \quad 1 5 0 \quad 2 0 0 \quad 2 5 0 \quad 3 0 0$

(b) Total length of the network links over the average value of this quantity

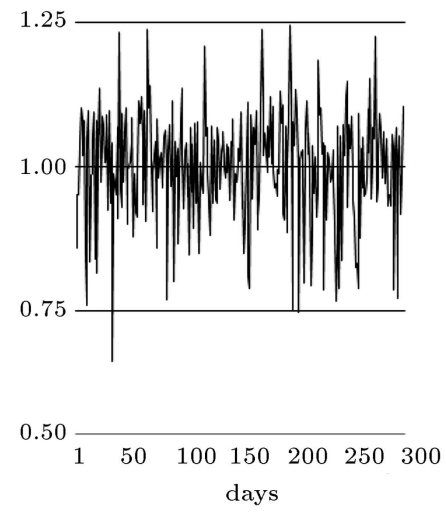

(c) Total maximum throughput over the average value of this measure

Figure 16. Large variation in throughput due to small variation in inputs (O/D demand and network length).

the network into a "chaotic" situation. Suppose the average car (equivalent) occupancy rate is $\mathrm{Co}_{e}$, the average number of trips per capita per day is $t$, and $f$ is the portion of the total daily vehicular trips of the city occurring in the morning peak hour. Then, we may write:

\section{( City population capacity from $)$}

(its transportation point of view)

$$
\begin{aligned}
& =\frac{\left[c O_{e}\left(\frac{\text { pass. trips }}{\text { pcu. trips }}\right) \cdot q_{\max }\left(\frac{\text { pcu. trips }}{\text { peak hour }}\right)\right]}{\left[t\left(\frac{\text { pass. trips }}{\text { persons. day }}\right) \cdot f\left(\frac{\left(\frac{\text { pass. trips }}{\text { peak hour }}\right)}{\left(\frac{\text { pass. trips }}{\text { day }}\right)}\right)\right]} \\
& =\frac{c O_{e} \cdot q_{\text {max }}}{t . f} \text { persons. }
\end{aligned}
$$

For the city of Mashhad, we have $c_{e}=3.65, t=1.6$, and $f=0.0772$, and from Figure 13, we estimate $q_{\text {max }}=127,978$. Then, from Eq. (26), we may compute city population capacity $\approx 3,800,000$. That is, to prevent "chaotic" traffic flow in the city (in the morning peak) with the current transportation technology, we should hold its population much below 3.8 million. Alternatively, we may invest on the network to increase its roadway network capacity (Figure 17) or take the demand off the road by encouraging car users to choose public transportation by suitable means.

\section{Conclusions}

This paper intends to convey the fact that there are threats to the resiliency of a large city. One important 


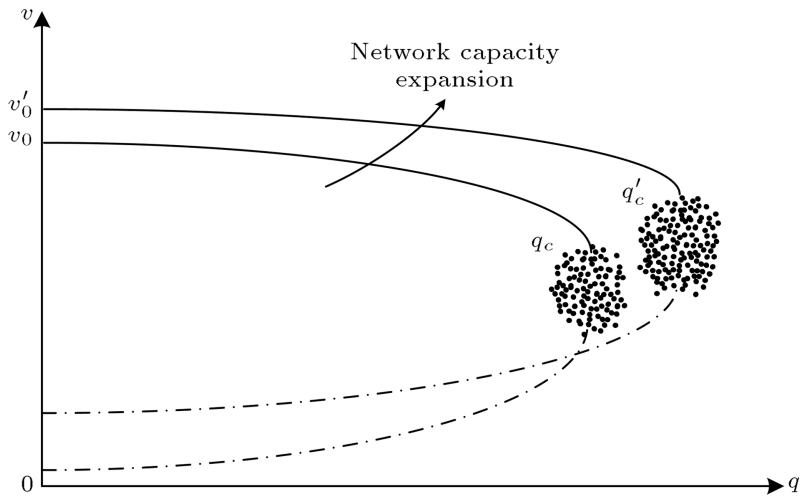

Figure 17. Demand management (wiser network use) and/or supply increase (network expansion) to avoid chaos in the network.

factor in this respect is the population capacity of the city. It is clear that a city has a population capacity from the different resources points of view: transportation, electricity, water, gas, communication, etc. Transportation-imposed capacity, however, is of particular importance, as it limits the activities and economics of the city. However, it is not clear that: What happens if the city passes this limit? How may we return the city to a stable situation (by, e.g., education, demand/supply management, or investments in supply?), when it turns into a chaotic one? What is the value of this limiting/critical capacity at the first place?

This paper is an attempt to analyze these questions and draw an answer for them. Section 2 tries to draw suitable transportation network performance conditions by employing an existing evolutionary model of traffic flow, to show how chaotic situations occur at certain demand and supply parameter values. Moreover, it shows a way to return the system back to its normal operation. This is done on a small expository network available in the literature.

Section 3 is an attempt to revisit the experiments done by Greenshields [34] in 1934 for an actual freeway section, but this time for a large real city. Under certain assumptions, it designs a simulation experiment and shows that the same phenomenon experienced by Greenshields occurs for this city: As demand increases (for all O/D's) from a minimum level, the average network travel speed $(v)$ decreases, while increasing the network throughput $(q)$ following a rather clear function, (until) up to a maximum level at which point the function ceases to exist, and a chaotictype (Greenshields' unstable) situation arises. Further increase in the demand decreases both the speed and the throughput of the network, but in a rather clear $V-q$ relationship. A large number of flow simulations at the demand levels in the region of unstable flow show that small changes in the demand and supply of the network result in large variations in the throughput of the network, such that the time series of the latter values to an external observer is chaotic (with positive Lyapunov exponent). The discussion in this section poses the question of the limit to the resiliency of the city from the standpoint of its transportation network capacity, and estimates a value for the maximum population of the city to prevent it from falling into the chaotic region of traffic flow. It is, then, argued how to take the network out of this situation.

\section{References}

1. "Resilient city", Available at: http://www.resilientcity.org. [Accessed Feb. 2014].

2. "The resilience alliance", Available at: http://www.resalliance.org.

3. Walker, B., Holling, C.S., Carpenter, S.R. and Kinzig, A. "Resilience, adaptability and transformability in social-ecological systems", Ecology and Society, 9(2), p. 5 (2004).

4. "Ward C (2007) diesel-driven bee slums and impotent turkeys: The case for resilience", Available at: http://www.theragblog.com/crisis-is-also-anopport unity-for-change/ [Accessed May 29] (2015).

5. Bumphus, S.D. Assessing Resiliency and Sustainability in an Intelligence Infrastructure System, Old Dominion University (2012).

6. Bie, J. and Lo, H.K. "Stability and attraction domains of traffic equilibria in a day-to-day dynamical system formulation", Transportation Research Part B: Methodological, 44(1), pp. 90-107 (2010).

7. Lu, S. and Nie, Y.M. "Stability of user-equilibrium route flow solutions for the traffic assignment problem", Transportation Research Part B: Methodological, 44(4), pp. 609-617 (2010).

8. Li, X., Peng, F. and Ouyang, Y. "Measurement and estimation of traffic oscillation properties", Transportation Research Part B: Methodological, 44(1), pp. 1-14 (2010).

9. Nie, Y.M. "Equilibrium analysis of macroscopic traffic oscillations", Transportation Research Part B: Methodological, 44(1), pp. 62-72 (2010).

10. Xia, Y., Liu, N. and Iu, H.H. "Oscillation and chaos in a deterministic traffic network", Chaos, Solitons \& Fractals, 42(3), pp. 1700-1704 (2009).

11. Cantarella, G.E. Fixed-Point Stability and Bifurcations in Dynamic Processes for Traffic Assignment, IOSS Press (1997).

12. Nagatani, T. "Chaos and dynamical transition of a single vehicle induced by traffic light and speedup", Physica A: Statistical Mechanics and its Applications, 348, pp. 561-571 (2005).

13. Nagatani, T. and Nagai, R. "Chaotic and periodic motions of two competing vehicles controlled by traffic lights", Chaos, Solitons \& Fractals, 25(1), pp. 245-253 (2005). 
14. Zhang, X. and Jarrett, D.F. "Chaos in a dynamic model of traffic flows in an origin-destination network", Chaos: An Interdisciplinary Journal of Nonlinear Science, 8(2), pp. 503-513 (1998).

15. Guo, R.-Y. and Huang, H.-J. "Chaos and bifurcation in dynamical evolution process of traffic assignment with flow "mutation"", Chaos, Solitons \& Fractals, 41(3), pp. 1150-1157 (2009).

16. Lan, L.W., Lin, F.-Y. and Kuo, A.Y. "Identification for chaotic phenomena in short-term traffic flows: A parsimony procedure with surrogate data", Journal of the Eastern Asia Society for Transportation Studies, 6, pp. 1518-1533 (2005).

17. Lin, F.-Y. and Lan, L.W. "Traffic flow analysis with different time scales", Journal of the Eastern Asia Society for Transportation Studies, 6, pp. 1624-1636 (2005).

18. Lan, L., Lin, F. and Wang, Y. "Self-organization phenomenon and the edge of chaos in traffic flow dynamics", Proceedings of the Eastern Asia Society for Transportation Studies, 4(1-2), pp. 574-582 (2003).

19. Xu, M. and Gao, Z. "Chaos in a dynamic model of urban transportation network flow based on user equilibrium states", Chaos, Solitons \& Fractals, 39(2), pp. 586-598 (2009).

20. Wang, J., Shi, Q. and Lu, H. "The study of shortterm traffic flow forecasting based on theory of chaos", Intelligent Vehicles Symposium, 2005. Proceedings. IEEE. IEEE (2005).

21. Rassafi, A.A., Poorzahedy, H. and Vaziri, M. "An alternative definition of sustainable development using stability and chaos theories", Sustainable Development, 14(1), pp. 62-71 (2006).

22. Khorgami, M., Sustainable Transportation in Urban Systems: Application of Mathematical Methods to Verify The Existence of Chaos, MS Thesis, Department of Civil Engineering, Sharif University of Technology, Tehran, Iran (2004).

23. Bie, J. The Dynamical System Approach to Traffic Assignment: The Attainability of Equilibrium and Its Application to Traffic System Management, Hong Kong Univ. of Sci. and Tech. (Hong Kong) (2008).

24. Jin, W.-L. "A dynamical system model of the traffic assignment problem", Transportation Research Part B: Methodological, 41(1), pp. 32-48 (2007).

25. Sheffi, Y., Urban Transportation Networks: Equilibrium Analysis with Mathematical Programming Methods, Prentice-Hall, Englewood Cliffs, NJ (1985).

26. Waller, S., Schofer, J. and Ziliaskopoulos, A. "Evaluation with traffic assignment under demand uncertainty", Transportation Research Record: Journal of the Transportation Research Board, 1771, pp. 69-74 (2001).

27. Ukkusuri, S.V., Mathew, T.V. and Waller, S.T. "Robust transportation network design under demand uncertainty", Computer Aided Civil and Infrastructure Engineering, 22(1), pp. 6-18 (2007).
28. Chen, A., Yang, H., Lo, H.K. and Tang, W.H. "Capacity reliability of a road network: an assessment methodology and numerical results", Transportation Research Part B: Methodological, 36(3), pp. 225-252 (2002).

29. Shahabi, M., Network Design to Alleviate Chaos in The Transportation Network Flows: Cases of Deterministic and Uncertain Supply, MS Thesis, Department of Civil Engineering, Sharif University of Technology, Tehran, Iran (2010).

30. Sharifi, M.S., Urban Transportation Network Design in Chaotic Conditions and Uncertainty in Travel Demand, MS Thesis, Department of Civil Engineering, Sharif University of Technology, Tehran, Iran (2010).

31. Spiess, H. and Florian, M. "Optimal strategies: a new assignment model for transit networks", Transportation Research Part B: Methodological, 23(2), pp. 83102 (1989).

32. INRO "EMME/2 user manual", Software Release 8, Montreal, Canada (1996).

33. ITSR: The Institute for Transportation Studies and Research, Traffic Demand Prediction and Assignment Model for the City of Mashhad, Mashhad Comprehensive Transportation Studies, Report No. 76-09, Sharif University of Technology, Tehran, Iran (in Farsi) (1994).

34. Greenshields, B. "A study of traffic capacity", Proceedings of the Highway Research Board, 14, TRB, Washington, D.C. (1934).

35. Chen, A., Yang, H., Lo, H.K. and Tang, W.H. "A capacity related reliability for transportation networks", Journal of Advanced Transportation, 33(2), pp. 183200 (1999).

36. Banks, J., Carson, J. and Nelson, B., Discrete-Event System Simulation, Prentice-Hall, Englewood Cliffs, NJ (1996).

37. McShane, W. and Roess, R., Traffic Engineering, Prentice-Hall, Englewood Cliffs, New Jersey (1990).

38. Moon, F.C., Chaotic and Tractal Dynamics: An Introduction For Applied Scientists and Engineers, John Wiley \& Sons, Inc., New York (1992).

39. Kapitaniak, T., Chaos for Engineers: Theory, Applications, and Control, Springer Science \& Business Media (2012).

40. Cambel, A.B., Applied Chaos Theory: A Paradigm for Complexity, Academic Press, Inc., London (1993).

41. Chen, Y. and Leung, A.Y.T., Bifurcation and Chaos in Engineering, Springer Science \& Business Media (2012).

42. Schuster, H.G. and Just, W., Deterministic Chaos: An Introduction, 4th Ed., Wiley-VCH, Weinheim, Germany (2005).

43. Xu, M. and Yang, J. "Analyzing nonlinear time series of multi-lane highway traffic volume", Journal of Physics: Conference Series, IOP Publishing (2008). 
44. Shang, P., Li, X. and Kamae, S. "Chaotic analysis of traffic time series", Chaos, Solitons \& Fractals, 25(1), pp. 121-128 (2005).

45. Best, E., Adami, T. and Zhu, J. "Instability detection for nonlinear time-varying systems using Lyapunov exponent", AIAA Guidance, Navigation, and Control Conference and Exhibit (2003).

\section{Biographies}

Mohammad Sadra Sharifi is a PhD candidate in the Department of Civil and Environmental Engineering at the Utah State University. He received his BSc degree in Civil Engineering from Ferdowsi University of Mashhad, Iran, in 2008, and MSc degree in Civil Engineering (Transportation Engineering) from Sharif University of Technology, Iran, in 2010. He served as a consultant engineer in Iran, where he worked on traffic operation and highway design projects. He is currently a research assistant working on projects related to pedestrian walking behavior modeling involving individuals with disabilities. His research interests include pedestrian behavior modeling, transportation planning, and travel behavior modeling.

Mehrdad Shahabi received the PhD degree in Transportation Engineering from West Virginia University, West Virginia, USA, in 2015. He is currently a Research Associate at University of Florida. His research interests include supply chain networks, mathematical programming, and transportation network modeling.

Elnaz Abshar received her MS degree in Transportation Engineering and Planning in 2006 from Sharif University of Technology. She has experience in statewide transportation planning as well as urban transportation planning. Her expertise includes transportation demand and network flow modeling.

Mohammad Hossein Khorgami (BSc, MSc, MCIHT) is currently Principal Consultant, Transportation, providing transport planning advice to both public and private sectors. He has worked on integrated transport planning and modelling covering the life cycle of projects from survey specification and data collection to the development and application of complex transport modelling tools to help assist and appraise the decision making process. He has worked in the management, development and application of demand forecasting models, economic appraisal and economic case development, multi-modal model projects, and discrete choice and activity-based demand modelling. He has undertaken transport planning projects in UK, Cyprus, Qatar, Kuwait, Malaysia, New Zealand, and Iran.

Hossain Poorzahedy is Professor of Transportation at Sharif University of Technology, Department of Civil Engineering. He received his $\mathrm{PhD}$ degree from Northwestern University (1980), MS degree from Stanford University (1976), and BS degree from Shiraz University (1975). His life-long interest has been in various aspects of transportation network design (solution methods; large real network case studies; network reliability, capacity, sustainability, resiliency, and chaotic behavior; network pricing; and network development finance through Build-Operate-Transfer schemes). His recent works bring together land use-transportation interaction, transit-oriented development, and disaster management as related to transportation. 